\title{
COVID-19 and Future Disease $X$ in Circular Economy Transition: Redesigning Pandemic Preparedness to Prevent a Global Disaster
}

\author{
Cristina Possas, et al. [full author details at the end of the article]
}

Received: 1 March 2021 / Accepted: 6 May 2021 / Published online: 28 June 2021

(C) Springer Nature Switzerland AG 2021

\begin{abstract}
The COVID-19 pandemic exposed a world surprisingly unprepared to respond to the new epidemiological scenario, even the developed countries, in spite of warnings from scientists since the 1990s. These alerts warned on the risks of an exponential increase in emergence of potentially pandemic zoonotic infectious diseases related to disruptive ecological niches in different regions of the globe, such as H1N1 Influenza, SARS, MERS, Zika, avian flu, swine flu, and Ebola, and also on the risks of a future and more lethal Disease X. We examine this global public health failure in anticipating and responding to the pandemic, stressing the urgent need for an innovative global pandemic preparedness system in the current transition from linear economy to a circular economy. Evidence provided here indicates that this novel preventive-based and resource-saving preparedness system could contribute to reverse the detrimental impacts of the pandemic on global economy and increase its resilience. Individual protection, contact tracing, and lockdown have proved to be just partially effective to respond to the spillover of viral zoonosis into the human population, and for most of these pathogens, vaccines are not yet available. As for COVID-19 vaccines, in spite of the extraordinary investments and unprecedented advances in innovative vaccines in few months, most of these products are expected to be available to more vulnerable developing countries' populations only by mid-2022. Furthermore, even when these vaccines are available, constraints such as low efficacy, waning immunity, new concerning COVID-19 variants, adverse events, and vaccine hesitancy might possibly restrict their public health impact and could contribute to aggravate the pandemic scenario. Considering these constraints and the severe global economic and social crises resulting from the lack of adequate preparedness and delayed effective response to COVID-19 and possibly to a future Disease X, we propose a proactive global eco-social pandemic preparedness system. This novel system, based on One Health paradigm and on artificial intelligence and machine learning, is expected to incorporate "spillover" foresight and management into global preparedness and timely response. Designed to mitigate damage from outbreaks and minimize human morbidity and mortality, this approach to pandemic foresight and preparedness will be key to prevent a global disaster.
\end{abstract}

Keywords COVID-19 $\cdot$ Disease X $\cdot$ Vaccines $\cdot$ Pandemic preparedness $\cdot$ Ecology $\cdot$ Circular economy 


\section{Introduction}

Conceptual incorporation of ecological and evolutionary approaches into public health is not new. Scientists have long warned that the rate of emergence of new infectious diseases is exponentially increasing [1-3] and the need for ecosystem and eco-social perspectives to deal with these new global epidemiological scenarios. Confirming these alerts, challenging outbreaks of high impact zoonotic infectious diseases have increased cumulatively from fewer than 1000 in 1980 to more than 3000 by 2010, with diseases such as influenza, severe acute respiratory syndrome (SARS), Middle East respiratory syndrome (MERS), Ebola, and Zika emerging in a new pandemic era [4-6]. In the past decade, aside from COVID-19, the World Health Organization (WHO) designated five other disease outbreaks as a "public health emergency of international concern": Ebola (twice), swine flu, poliovirus, and Zika. Some of these alerts on emergence of coronaviruses were surprisingly accurate: in 2017, a scenario study from the Johns Hopkins Center for Health Security [7] anticipated for year 2025 a new coronavirus pandemic, which they had named SPARS-CoV-1. Several other publications had since equally alerted for the increasing risks in a near future of a coronavirus pandemic resulting from spillover from bats to humans. Moreover, there is a consensus in the scientific community that COVID-19 may not disappear but persist as an endemic disease with a seasonal pattern or may cause new waves of serious illness, as seen during the Spanish flu in 1918-1920. In the past centuries, there were several pandemic diseases. The Spanish flu infected 500 million people, about one-third of the world's population at the time, in four successive waves, with a death toll estimated in 50 million, in one of the deadliest pandemics in human history [8]. We present a historical perspective of these main zoonotic epidemics and pandemics since the beginning of the last century. Is the world prepared to face the next COVID-19 pandemic, avoiding and mitigating its potentially severe consequences? Or a future and more lethal new Disease $\mathrm{X}$ ? The answer is certainly no. The current COVID-19 pandemic exposed a world surprisingly unprepared, even the developed countries, revealing the current constraints of national health systems: inadequate information and surveillance systems to support immediate response; insufficient and unqualified medical and nursing staff faced with perplexity in dealing with massive demand for intensive care in hospitals; and lack of basic equipment for individual and collective protection, limitations which have certainly favored the accelerated global dissemination of SARS-CoV-2. Furthermore, these health system constraints have been amplified by limited knowledge and health education, even in developed countries, on basic preventive procedures for infectious pathogens, such as hand washing. There is therefore an urgent need for a paradigmatic change in global preparedness and response to emerging and resurgent infectious diseases, particularly the high-risk zoonotic ones. This new approach from governments and international organizations should be able to intervene and act more effectively in order to minimize zoonotic spillover and to mitigate the damage of new pandemic diseases. An eco-social framework for a new global preparedness system is proposed, integrating foresight, surveillance, and response, supported by a new informational paradigm — artificial intelligence algorithms, machine learning, and extensive databases ("big data"), directed to prevent and manage zoonotic pathogen spillover from wildlife reservoirs into human and livestock populations. This novel preparedness paradigm might contribute to drastically reduce the massive economic and social impacts of pandemics and contribute to global economy resilience, in the circular economy transition. 


\section{Conceptual Issues: Pandemic Preparedness in Circular Economy Transition}

The COVID-19 pandemic emerged in a global context of international debate on the negative impacts of a linear economy and on the urgent need for a transition to a resource-saving circular economy by 2050, dissociating economic growth and social development from extensive resource use and environmental impact, as recently illustrated by the European Union Circular Economy Action Plan Eurasia Group [9]. The huge impact of the pandemic on global economy has resulted in international calls for recovery, aligned with diverse environmental, social, and health challenges, as indicated in a recent report on a global COVID-19 recovery strategy, conceived to minimize these impacts [10]. In order to achieve these goals, governments have been pressed to take critical actions in diverse areas, aiming to protect their national economies from the pandemic, reducing their vulnerability, and laying the foundations for a more resilient global economy. The circular economy transition will thus contribute to accelerate this economic recovery, opening a window of opportunity for a novel resourcesaving eco-social pandemic preparedness system, as presented here. From this perspective, the major challenges in the health sector are how to overcome the constraints of traditional, ineffective, and resource-consuming public health approaches and rapidly put in place a comprehensive and effective global eco-social preparedness system, incorporating vaccine preparedness. In contrast with the US\$10 trillion economic stimulus to recovery from COVID pandemic announced by 54 governments $[10,11]$, very low funding and incentives have been so far provided to support global preparedness and response. This new global preparedness paradigm represents therefore a systemic shift that can certainly contribute to accelerate recovery and to increase global economy resilience to pandemics, providing environmental and societal benefits and saving lives.

\section{Public Health Failure: Exponential Pandemic Costs}

Considering the long global history of epidemics and pandemics and all alerts by the scientific community and stakeholders, why governments and international authorities were caught by surprise in COVID-19 pandemic and were so late in response? Failure to adequately anticipate and manage the pandemic resulted in a dramatic global crisis, with severe social and economic impacts, evidencing gaps and limited national health systems' capacity to prevent, detect, and respond to emerging diseases outbreaks, both in developed and developing countries. The lack of adequate investment in innovative vaccine development and preparedness, surveillance, and response to the COVID-19 pandemic has already resulted in huge global economic impact. In the USA alone, this economic impact is estimated to result in a total cost of US\$ 16 trillion, corresponding to $90 \%$ of the country's GDP [12]. This enormous impact contrasts with very low global investments in vaccines and global preparedness. This contrast is evidenced by a recent report by the Eurasia Group commissioned by the Bill \& Melinda Gates Foundation, highlighting that the economic benefits of a global equitable vaccine solution alone for the 10 developed countries included in the analysis (Canada, France, Germany, Japan, Qatar, South Korea, Sweden, United Arab Emirates, the UK, and the USA) would be at least US\$ 153 billion in 2020-2021, rising to US\$ 466 billion by 2025. This is more than 12 times the US\$ 38 billion estimated total cost of the Access to Covid Tools (ACT) Accelerator launched by the World Health Organization and partners Eurasia Group [9]. In other words, besides the cost in 
lives, these insufficient investments in vaccines and pandemic preparedness also cost money. The COVID-19 pandemic increased the global visibility of vaccine policy as a critical component of economic policy and contributed to the recognition by international organizations such as the International Monetary Fund (IMF) that accelerating vaccines and other preventive measures to end the health crisis could add nearly US\$ 9 trillion to the global GDP by 2025 [13]. In spite of this recognition, it should be noted that the current pharmaceutical business model does not provide incentives and awards for investments in the development of vaccines and other preventive technologies. In fact, this business model is fundamentally designed to reward treatments, not prevention. Thus, novel funding and incentive mechanisms for prevention should be urgently conceived. Vaccine investments should not be remunerated on a production cost basis: the remuneration should be proportional to the tangible and intangible values that vaccines provide. Several international expert panels have recommended in the last two decades specific reforms for outbreak and pandemic preparedness, including vaccine preparedness, but gaps and weaknesses remain $[4,5]$. There have been plenty of global initiatives in this direction, but their impact has been very low. International political bodies, such as the G7, G20, G77, and several regional intergovernmental organizations have adopted political commitments for action on various aspects of health and health emergencies, including funding and linkages to health system strengthening and universal health coverage. In spite of their important contributions, most of these initiatives have been public health-oriented and limited in their scope. They often miss the ecological and evolutionary perspectives and tools to anticipate zoonotic-related pandemics, often intervening too late, long after the occurrence of spillovers from high-risk reservoirs. The current infection disease surveillance system, which was conceived and implemented in the 1950s, is inadequate for preparedness and response to emerging infectious disease outbreaks and pandemics. It is expensive and has very low sensitivity and specificity. The surveillance system needs to be conceived and implemented from a new paradigm, including effective vaccine preparedness, and with significant capital investments to incorporate novel technologies that can reduce costs and increase its sensitivity and specificity. Participative surveillance methods should be incorporated into this novel surveillance system in order to assure that the population can actively contribute to surveillance. Furthermore, an evolutionary ecological perspective is often missed in public health and epidemiological surveillance, and the absence of this perspective might explain previous failures in dealing with unexpected pandemics. The complex conditions affecting the evolution, introduction, mutations, variations, and adaptations of zoonotic pathogens in new ecological niches should be understood from this perspective ([1], Lewontin, 1974, [14]). A variational approach to evolution (Lewontin, 1974) is crucial for understanding disease emergence, because it provides insights into the evolutionary changes in vectors, pathogens, and diseases Levins et al. [1]. Additionally, it is important to understand that evolution is not progressive; rather, it occurs in opportunistic and unpredictable ways. The notions that usually prevail in global public health and epidemiological surveillance approaches include equilibrium-oriented points of view, assumptions of linearity, and teleological perspectives. However, these notions must be overcome to deal with disease evolution in complex ecosystems and with unpredictable evolutionary processes in general Levins et al. [1]. In the following topics, we discuss the advances and limitations of public health interventions in dealing with pandemics and how global preparedness should be redesigned to meet these new challenges. 


\section{COVID-19 Vaccines: Breakthroughs and Constraints}

Vaccines are internationally recognized as the most cost-effective and resource-saving public health strategy. Vaccine policy emerged in the COVID-19 pandemic as a powerful economic policy, critical for global economic recovery. The rapid dissemination of SARS-CoV-2 coronavirus worldwide has exponentially increased international expectations to accelerate the development of vaccines. The international collaborations have already succeeded to bring into the market innovative and high-efficacy mRNA vaccines (Moderna, Pfizer/BioNTech), engineered vector vaccines (Oxford/AstraZeneca - ChAdOx1), and several other vaccines with diverse technologies. Although evidence supported by quality data is still needed, there are indications that these vaccines have already contributed to significantly reduce COVID-19 morbidity and mortality in several countries, particularly in the more vulnerable elderly populations (Wise, 2021). The global efforts to develop a COVID-19 vaccine have been extraordinary and unprecedented. Never in the history of vaccine development, so many governments, public and private research institutes, and public and private funds have been involved in collaborative initiatives providing, in less than 9 months from the emergence of the pandemic in Wuhan, China, huge investments for the development of a vaccine. This scenario has led to an unparalleled accelerated international search for a vaccine, with 195 projects worldwide, with 10 in clinical phase 3, 34 in phase 1/2, and 151 in pre-clinical stage [15]. This accelerated speed in the development of innovative vaccines such as mRNA vaccines (Moderna, Pfizer/BioNTech) and engineered vector vaccine (Oxford/AstraZeneca, ChAdOx1 vector) benefited from previous developments from other emerging diseases vaccines, such as SARS and Zika vaccines. Considering that data for these vaccines will be obtained at a very fast speed, there will be issues related to vaccine safety, which will probably require 6-12 months' evaluation or even longer, even with "fast track" procedures. The decision to go ahead with new COVID-19 vaccine immunization under these unassessed safety conditions has been a very difficult and controversial decision, justified only by the severity of the pandemic. It will be necessary to organize a highly qualified pharmacovigilance group, in order to identify the rate of adverse events potentially expected for these vaccines, which can be detected only after millions of doses are applied. Several other interventions have also been put in place in many countries to mitigate the SARS-CoV-2 expansion and flatten the peak of cases, such as lockdown, social distancing, use of mask, and disinfection. Notwithstanding the relevance of these interventions, there is a consensus in the scientific literature that the most important instrument to control virus spread in human populations is reaching herd immunity, via infection or via vaccines. Vaccines are considered the most important strategy to achieve herd immunity. Natural herd immunity via infection should never be a policy, since it is ethical unacceptable to let people become infected by a virus on which we have very little knowledge on its possible sequelae and long-term pathologies. Natural herd immunity just refers to an equilibrium between virus and host, a default mechanism for achieving interruption of viral transmission [16]. Considering that today's global number of COVID-19 cases, even when multiplied by 10 , considering both asymptomatic and under-reported cases, accounts for around $0.5 \%$ of the world population, in order to reach the required populational threshold for herd immunity, it would be necessary to have many more people infected by SARS-CoV-2 or to vaccinate at least $75 \%$ of the global population. A recent publication [17] argues there is little evidence to suggest that the spread of SARS-CoV-2 might stop naturally before at least $50 \%$ of the population has become immune, in opposition to previous mathematical modeling studies indicating that this threshold for herd immunity from natural infection could be much 
lower, from 10 to $20 \%$ [18]. In addition, it should be also noted that besides developing effective new COVID-19 vaccines, it is imperative to achieve a long duration of immune protection from these vaccines. Nevertheless, concerning the duration of protection, an important scientific controversy has emerged on waning immunity. There is a possibility that we may never reach herd immunity from SARS-CoV-2 infections or vaccines since, differently from many other viruses, the immunity conferred by this virus would be temporary, lasting nearly 6 months and then fading, with a possible risk of reinfection. As noted by a recent study [19], this transient neutralizing antibody (nAb) response is a feature shared by both a SARS-CoV-2 infection that causes low disease severity and the circulating seasonal coronaviruses that are associated with common colds. Notwithstanding, in recent studies, other authors are more optimistic and argue that even with the immune response from antibodies dropping to vanishingly low levels a few months after the viral infection, it is necessary to recognize that immunity does not rely entirely on antibodies but also on $\mathrm{T}$ cell response [20, 21 ] and that memory cells would react fast enough to contain the infection and prevent severe disease. In addition, a major challenge are the concerning recent SARS-CoV-2 variants (UK, B.1.1.7; South Africa, B.1.351; and Brazil P1), requiring from scientists a redesign of approved vaccines. Finally, other issues such as limited global access to multipatented vaccines [22], sharp decline in global vaccine coverage, related to "vaccine hesitancy," and vaccine refusal [23] will probably contribute as well to reduce the impact of the new SARS$\mathrm{CoV}-2$ vaccines. In order to meet global challenges in vaccine development for emerging diseases, a new vaccine preparedness initiative has been created in 2017 by governments and international organizations. Germany, India, Japan, Norway, the Bill \& Melinda Gates Foundation, the Wellcome Trust, and the World Economic Forum founded the strategic Coalition for Epidemic Preparedness Innovations (CEPI) to facilitate focused support for vaccine development to combat major health epidemic/pandemic threats. CEPI introduced extraordinary advances in global vaccine development for COVID-19 and other potentially pandemic diseases and should be urgently strengthened, with its funding and incentives exponentially increased in a sustainable way to adequately respond to future pandemic scenarios.

\section{Preventing Zoonotic Spillover: Future Disease X}

An overview of the main emerging pandemic and epidemic human diseases from 1918 to 2020 and related indicators is presented in Table 1. Interestingly; all of them are of zoonotic transmission: bats were reservoirs for four of these nine diseases (Ebola, MERS, SARS, COVID-19), birds for two of them (Spanish flu, Asian avian influenza), chimpanzee for one of them (HIV/AIDS), Aedes mosquitos to one of them (Zika), and pigs to one of them (Swine influenza). Three of these diseases resulted in pandemics with major impact on global economies and societies: Spanish flu, ended in 1920 with 500 million cases (one-third of global population) and 50 million deaths; HIV/AIDS, ongoing, with 76 million cases and 33 million deaths; and COVID-19, ongoing, with 40.728 .371 cases and 1 million deaths, as of October 20, 2020 Johns Hopkins University [26]. An important lesson can be learned from these pandemics: until national health systems can provide early identification and control of high-risk pathogens at source, governments and international authorities will continue to respond to outbreaks of emerging disease late, long after epizootics such as COVID-19 have spilled over into human populations [30]. Two pandemics are ongoing, COVID-19 [31] and HIV/AIDS [24, 25]; Sharp et al., 2011; Keele et al., 2006), and one pandemic preparedness is 


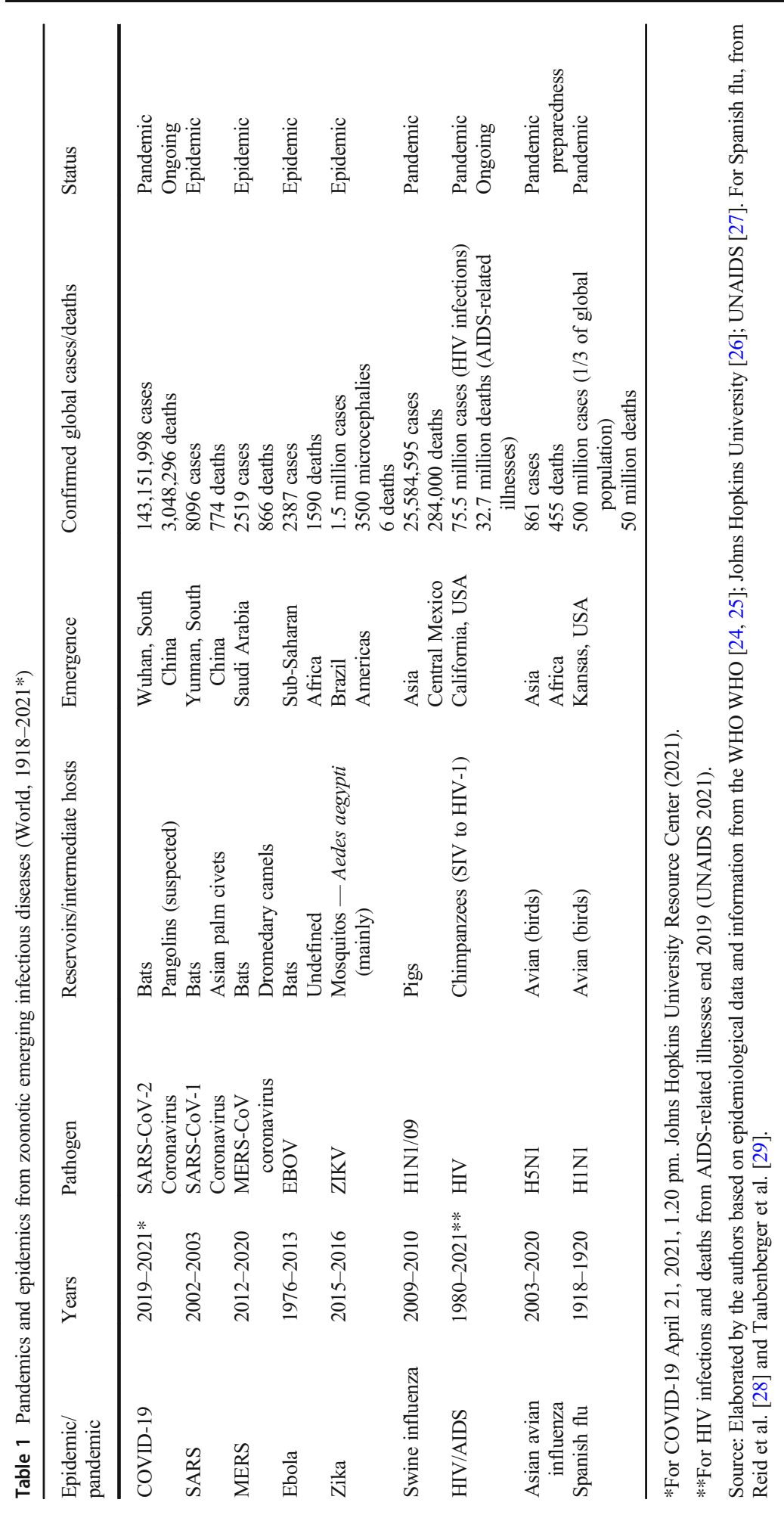


in place for highly pathogenic Asian avian influenza $\mathrm{H} 5 \mathrm{~N} 1$ [25, 32, 33]. Zika, transmitted by Aedes mosquitos, resulted in an epidemic with 1.5 million cases and 3500 microcephalies in newborns in Brazil and other countries in the American region (de Araújo et al., 2016; [34-36]. Swine influenza pandemic H1N1 which occurred in 2009-2010 [25, 33, 37] is also subject to monitoring and preparedness for a highly pathogenic strain [25, 33]; Ebola [38, 39], MERS [40, 41], and SARS [42-44] have also resulted in epidemics, raising international concerns for their pandemic potential.

In Fig. 1 a picture of viral spillover for these nine pandemic/ epidemic diseases is provided, indicating that they have resulted from human contact with five categories of reservoirs: bats, birds (poultry and wild), pig, chimpanzee and Aedes mosquitos in different emergence areas. There are many other high-risk viruses in nature that could potentially result in a future disease $\mathrm{X}$ pandemic. Scientists have recently developed a risk ranking framework supported by an interactive web tool, SpillOver, that estimates a risk score for wildlife-origin viruses, creating a comparative risk assessment of viruses with uncharacterized zoonotic spillover potential alongside those already known to be zoonotic [45]. Using data from testing 509,721 samples from 74,635 animals as part of a virus discovery project and public records of virus detections around the world, they succeeded to rank the spillover potential of 887 wildlife viruses.

There are over 250 viruses that have already jumped from animals to humans and resulted in pandemics: SARS-CoV-2 is the latest but certainly other not the last. Scientists estimate that there are about 1.7 million undescribed animal viruses, half of which have the potential to spill over into humans and could result in a future Disease X pandemic. Some examples of highrisk viruses are Ebola, Lassa virus, Seoul virus, and Nipah, a virus that has already infected humans, with annual spillover events.

Scientists should be able to identify potential locations for spillover in environmentally disturbed areas, supporting timely and more effective response. Finally, it is important to note that once spillover occurs, the epidemics disseminate rapidly into insalubrious urban areas worldwide, and disease prevention measures should be incorporated into these areas to

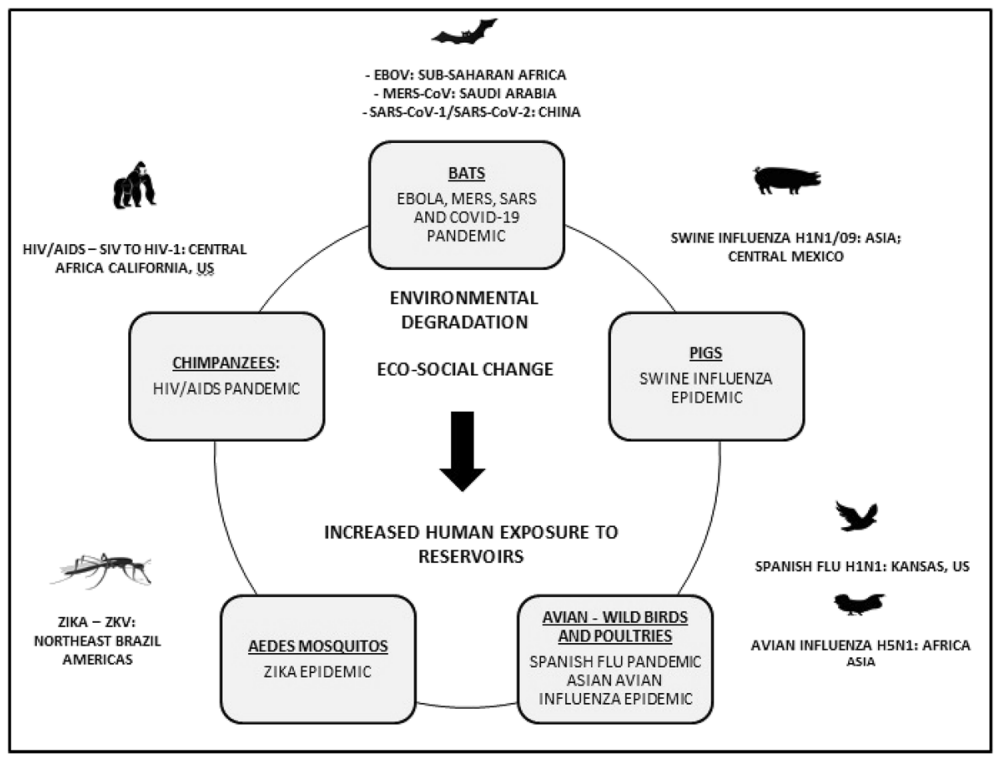

Fig. 1 Reservoirs and regions of emergence of pandemics and epidemic. Source: elaborated by the authors 
mitigate disease transmission. Improving the quality of data and identifying these high-risk pathogens and reservoirs at source and innovative artificial intelligence and machine learning strategies will be key to allow an entirely new approach to the prediction of these emerging zoonotic diseases.

\section{Environmental Protection: Minimizing Human Exposure to Reservoirs}

Recent ecological studies are providing evidence supporting that environmental protection reducing human exposure to reservoirs is key to prevent zoonotic spillover and could have significant global economic and social impact Nature Ecology and Evolution Editor [46]. In one of these studies [47], the authors indicate that zoonotic host diversity increases in humandominated ecosystems, with increase in the populations of bats, rodents, and birds resulting from habitat destruction possibly leading to the increase in viral spillover from these animals to humans. The recent and controversial decision of the Danish government to kill 15 million minks, the total mink population in the country farms, due to a coronavirus mutation, in a delayed response after the spillover has occurred, evidences the complexity of these animalhuman viral passages in the transmission of SARS-CoV-2 in mink farms [48]. Environmental protection, minimizing habitat disturbance, is thus key to reduce human exposure to zoonotic spillover. A study has evaluated the economics of preventing zoonoses through reducing wildlife disturbance (Dobson et al., 2020) and contrasted estimated expenditure to prevent deforestation, reduce and regulate wildlife trade, and monitor disease emergence with the global cost of the pandemic to national economies. In nearly all scenarios, the cost of prevention over many years is very small when compared with the severe economic and social damage caused by a single pandemic such as COVID-19. We advocate for a redirection of these global efforts and investments into an innovative global pandemic preparedness system, aiming to integrate eco-social foresight and response to emerging pathogens. This system, designed to minimize or prevent spillover of these potentially pathogens into humans, could provide immediate early response to these threats.

\section{Reservoirs: Feasibility of "Self-Disseminating Vaccines"}

The delayed public health response to spillover from reservoirs to humans indicates that novel and more pro-active immunization strategies should be considered, such as vaccination directed to high-risk reservoir species, using innovative vector platforms. According to [49], these vaccines would aim to eliminate the threat of spillover before it occurs using "selfdisseminating vaccines" capable of autonomously spreading through wild animal reservoirs, targeting a wide range of human pathogens. The controversy on feasibility of this strategy was raised by considerations on the constraints of the earliest self-disseminating vaccine strategy, due to the possibility of reversion resulting from evolutionary and immunity processes. This earliest vaccine strategy had initially been designed to target two highly lethal rabbit-specific emerging infectious diseases in the European rabbit population, myxoma virus (MV) and rabbit hemorrhagic disease virus (RHDV) Murphy et al. [50]. Notwithstanding, new breakthroughs were incorporated into this strategy, and recent self-disseminating vaccine approaches have used cytomegalovirus (CMV), which is a beta-herpesvirus, as the disseminating vaccine platform. CMV infection is normally benign in the healthy host. This 
important difference removes the need to use attenuated strains. Novel recombinant vaccines, as noted by Nuismer [49], are thus a priori the most promising approach for a transmissible vaccine for reservoir species. The choice between attenuated and recombinant designs may determine how well the vaccine is able to self-disseminate. The authors argue that attenuated transmissible vaccines are unlikely to ever be suitable tools for eliminating human pathogens from their wildlife reservoirs. Effective transmissible vaccines developed using attenuation must maintain considerable levels of transmission while producing minimal disease. The authors conclude that although new methods of attenuation greatly limit evolutionary reversion, an attenuated vaccine whose R0 exceeds 1 will always pose an ongoing threat of reversion if the wild-type pathogen has been extinguished, enabling the attenuated virus to persist indefinitely. In conclusion, in spite of pending scientific and technological issues related to immunity and safety, this new self-disseminating vaccine approach should be further developed. If proved successful, this vaccine strategy might be in the future a valuable tool contributing to eliminate the threat of pathogenic spillover from animals to humans.

\section{Artificial Intelligence: Prediction and Response to Zoonotic Outbreaks and Pandemics}

Research breakthroughs in bioinformatics and "big data" analytics architecture and tools have contributed to extraordinary advances in diverse fields of human activity [51]. Canadian startup artificial intelligence (AI) company, BlueDot, benefiting from these advances, succeeded to anticipate COVID-19 outbreak in China before it had been identified by international organizations and governments, alerting by December 2019 its private sector and government clients that a cluster of "unusual pneumonia" cases were happening around a market in Wuhan, China [52]. Other similar AI initiatives are ongoing with another company BioSymetrics, in collaboration with Janssen and Sema4, developing strategies for the prediction of COVID-19 [53]. Another company, Biovista, has also made important advances in COVID-19 research, directed to the identification of bioactive compounds and future healthcare scenarios for the pandemic [54]. Notwithstanding their breakthroughs, these anticipatory AI strategies have not so far been capable to support policy makers to design and conceive an immediate and focused response to disease emergence. In addition, these AI strategies are still limited in their scope, and some authors suggest that due to limitations in current data gathering and quality necessary to AI foresight, it remains unclear to what extent it will be possible to anticipate novel pathogens likely to emerge in the near future. Naudé [55] stresses that in spite of extraordinary recent developments, AI has not yet been impactful against COVID-19, since its use has been hampered on one hand by lack of data and on the other by too much data. Overcoming these constraints for pandemic preparedness will require the recognition of these limitations and a new approach to viral spillover detection, outbreak anticipation, and immediate response. There is plenty of information from publications and data on well-known viruses, such as coronaviruses and other respiratory viruses with an established history of spillover and emergence into the human population that should be more adequately incorporated into novel AI strategies. The contributions of international initiatives aiming to improve the quality of data on emerging pathogens, high-risk reservoirs, and disturbed environments should be urgently incorporated into "big data" supporting AI foresight and response strategies. A good example is the PREDICT Project, based at the One Health Institute, University of California, Davis. This project is the world's largest effort to track down emerging zoonotic diseases and 
is spread over more than 30 countries. PREDICT seeks to identify the viruses or other microorganisms found in wild animals with the goal of predicting where the next zoonotic outbreak could occur. The project has analyzed samples from over 56,000 wild animals, including non-human primates, bats, rodents, and other wild species used in the bush meat trade. So far, over 800 novel viruses that have the potential to infect humans have been identified, half of which were found in bats, 234 in non-human primates, and 143 in rodents and shrews [56].

\section{Pandemic Preparedness in Circular Economy Transition: Al-Based Surveillance}

Adequate and timely response to emerging infectious pathogens and zoonotic diseases periodically spilling over into humans will require an entirely new approach to epidemiological surveillance in the transition from a linear economy to a resource-saving circular economy: an integrated eco-social surveillance system capable to anticipate, monitor, and rapidly intervene, integrating all currently fragmented and disperse public health and ecological strategies for foresight and intervention, as indicated in Fig. 2. Conceived from a One Health perspective, this framework for eco-social preparedness system for a future "Disease X" would be permeated by artificial intelligence, machine learning, and "big data" at all levels of operation.

A new global governance paradigm, integrating public and private in innovative funding mechanisms and incentives for researchers and field workers, will be key for the success of this approach to response to potentially pandemic zoonotic pathogens. The new eco-social pandemic preparedness system should be based on creating and/or strengthening the following 6 main components, assuring their coordination and operational feasibility, as indicated in Fig. 3:

1. Epizootic surveillance: identification of high-risk reservoirs and potentially vulnerable "spillover" areas, preventing or minimizing human exposure.

2. "Big data" and artificial intelligence supported by PREDICT Project and similar viral sampling initiatives in wild animals.

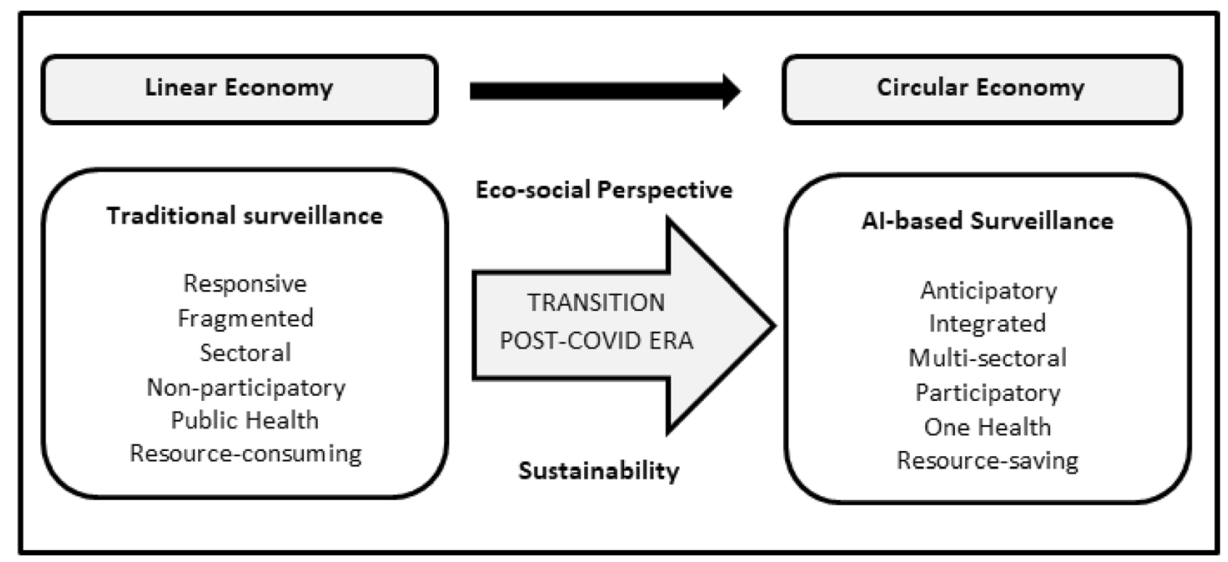

Fig. 2 Pandemic preparedness: new paradigm for surveillance in circular bioeconomy. Source: elaborated by the authors 
3. Syndromic surveillance: identification of syndromes characteristics of potential pandemics for the early detection and reporting of unusual diseases and events.

4. Genomic surveillance: routine genomic sequencing for SARS-CoV-2 and other potentially pandemic viruses. Important in a scenario of emergence of concerning viral variants in order to increase global availability of genetic sequence. This strategy is critical to support the development of diagnostic protocols and novel vaccines and to better understand the evolution and molecular epidemiology of these viruses.

5. Laboratory surveillance: analysis of samples for detection of unusual increases of pathogens.

6. Participatory surveillance: community participation of surveillance through direct access to self-diagnostic tools based on symptoms and self-administered diagnostic tests that are linked via cloud to a central AI data analyzes center.

\section{Conclusion}

Our results indicate that the main challenge for pandemic preparedness in the circular economy transition resides in the global ability of international agencies and governments to coordinate and immediately put in place effective foresight and preventive tools and interventions, supported by sustainable funding and intersectoral approaches. A critical issue, besides the urgent need to accelerate vaccine preparedness and universal access to immunization, is the necessity to strengthen the global governance capacity to anticipate and respond to high-risk zoonotic pathogens in different regions of the globe. These interventions should be able to assess these pathogens, to identify ecological niches for reservoirs and intermediate zoonotic hosts and immediately put in place spillover prevention, before a pathogen reaches the human

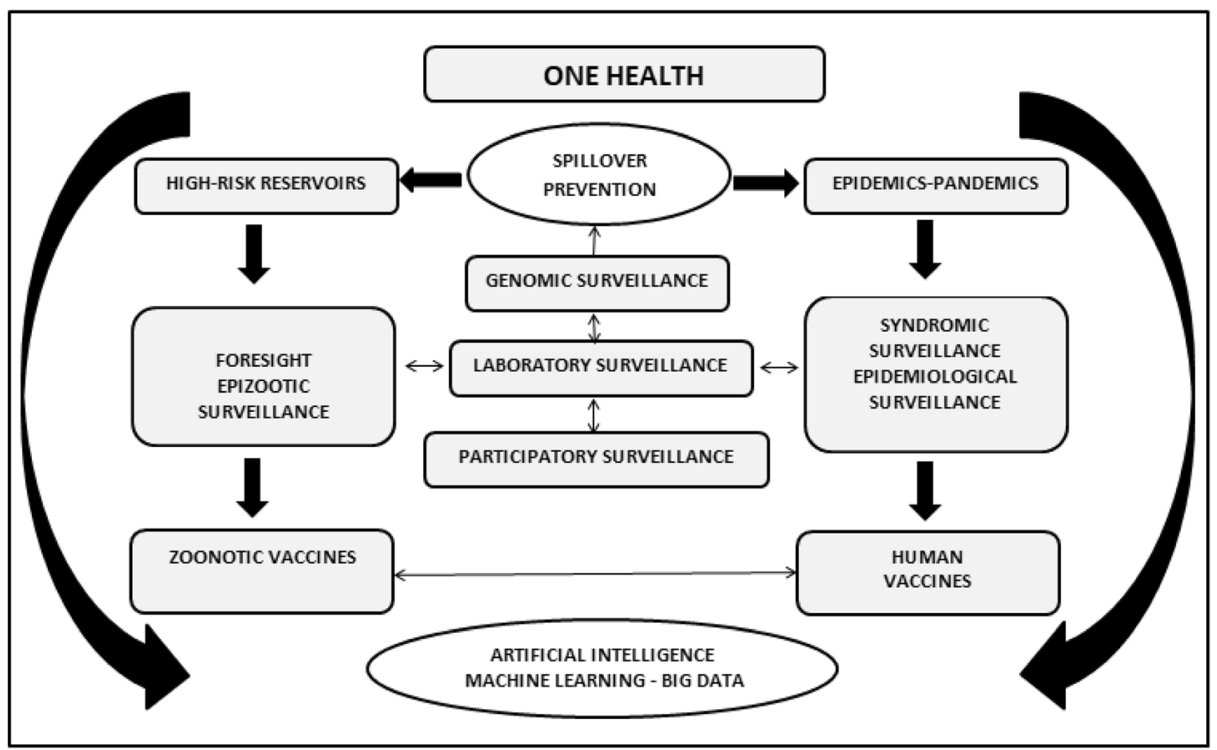

Fig. 3 Towards a new global eco-social pandemic preparedness system. Source: elaborated by the authors 
population and disseminates into epidemics or pandemics. If spillover has already occurred, foresight, epidemiological and zoonotic surveillance, and immediate preventive interventions should be implemented to minimize social and economic impacts of outbreaks. We provided evidence here indicating that public health failure in implementing a global preparedness system has already resulted in unprecedented economic crisis, with an exponential increase in COVID-19 pandemic costs estimated in 16 trillion US dollars, besides 10 trillion US dollars in economic stimulus for recovery by governments. Although pandemics like COVID-19, HIV/ AIDS, and the Spanish flu have been historically scarce, this scenario is rapidly changing as a result of environmental degradation and eco-social change. New outbreaks from zoonotic infectious diseases with pandemic potential have exponentially increased cumulatively from fewer than 1000 in 1980 to more than 3000 by 2010. These outbreaks are becoming more frequent with huge potential impacts on the global economy. Urbanization and deforestation are bringing humans and animals into closer contact worldwide, favoring intensification of spillover of emerging pathogens from animals into humans, with critical economic and social implications. This scenario is illustrated by the recent aggravation of COVID-19 pandemic by enhanced transmission of SARS-CoV-2 concerning variants in the UK (B.1.1.7), South Africa (B.1.351), and Brazil (P1). A paradigmatic change in public health is thus urgently needed to incorporate, from One Health perspective, the contributions of ecology and evolutionary theory not only conceptually but adequately translated into operational preparedness strategies and timely response. Vaccine preparedness, accelerating development, production, and universal access to immunization, supported by sustainable funding, is crucial to assure a sharp reduction in morbidity and mortality from COVID-19 and other potentially pandemic viruses. However, notwithstanding their utmost relevance and extraordinary health impacts, vaccines are not sufficient, since they are just a component of a necessary comprehensive global preparedness system. This novel One Health approach, supported by combined strategies, based on foresight, eco-social surveillance, artificial intelligence, machine learning, and "big data" analytical architecture will hence be key to improve global preparedness for zoonotic outbreaks and pandemics. From this perspective, the implementation of a pro-active and integrated global eco-social pandemic preparedness system will be critical to minimize the huge economic and social impacts of COVID-19 and of a possible future Disease X pandemic, laying the foundations for a more resilient and resource-saving global economy.

Code Availability Not applicable.

Author Contributions All authors contributed to the study conception and design. Material preparation, data collection, and analysis were performed by Cristina Possas and Akira Homma. The first draft of the manuscript was written by Cristina Possas and Akira Homma, and all authors commented on previous versions of the manuscript. All authors read and approved the final manuscript.

Data Availability Not applicable.

\section{Declarations}

Ethics Approval Not applicable.

Consent to Participate Not applicable. 
Consent for Publication Not applicable.

Conflict of interest The authors declare no competing interests.

\section{References}

1. Levins R, Awerbuch T, Brinkmann U, Eckardt I, Epstein P, Makhoul N, Possas C, Puccia C, Spielman A, Wilson ME (1994) The emergence of new diseases. Am Sci 82(1):52-60

2. Morse SS, Mazet JAK, Woolhouse M, Parrish CR, Carroll D, Karesh WB, Zambrana-Torrelio C, Lipkin WI, Daszak P (2012) Prediction and prevention of the next pandemic zoonosis. Lancet 380:1956-1965

3. Morse SS (1995) Factors in the emergence of infectious diseases. Emerg Infect Dis 1(1):7-15.1

4. GPMB (2019) Global Preparedness Monitoring Board. Annual Report A world at risk Geneva

5. GPMB (2020) Global Preparedness Monitoring Board. Annual Report A world in crisis Geneva

6. Wang LF, Crameri G (2014) Emerging zoonotic viral diseases. Rev Sci Tech Off Int Epiz 33(2):569-581

7. Schoch-Spana M, Brunson EK, Shearer MP, Ravi S, Sell TK, Chandler H, Gronvall GK. (2017) The SPARS pandemic 2025-2028: a futuristic scenario for public health risk communicators. Baltimore MD Johns Hopkins Center for Health Security

8. Spreeuwenberg P, Kroneman M, Paget J (2018) Reassessing the global mortality burden of the 1918 influenza pandemic. Am J Epidemiol 187(12):2561-2567

9. Eurasia Group (2020) Ending the covid-19 pandemic: the need for a global approach. Commissioned by The Bill \& Melinda Gates Foundation New York, NY

10. Ellen MacArthur Foundation (2020) The circular economy: a transformative COVID-19 recovery strategy. How policy can pave the way to a low carbon, prosperous future. https:/www.ellenmacarthurfoundation. org/assets/downloads/The-circular-economy-a-transformative-Covid19-recovery-strategy.pdf access April 18,2021

11. Cassim Z, Handjiski B, Schubert J, Zouaoui Y (2020) Public sector practice: the $\$ 10$ trillion rescue: how governments can deliver impact. McKinsey and Co

12. Cutler DM, Summers LH (2020) The COVID-19 pandemic and the \$16 trillion virus. JAMA 324(15): 1495-1496

13. Georgieva K. (2021) Transcript of International Monetary Fund Managing Director Kristalina Georgieva's Opening Press Conference Spring Meetings, April 7. https://www.imf.org/en/News/Articles/2021/04/07/ tr040721-transcript-of-imf-md-kristalina-georgievas-opening-press-conference-2021-spring-meetings Access April 10

14. Possas CA (2001) Social ecosystem health: confronting the complexity and emergence of infectious diseases. Reports in Public Health 17:31-41

15. WHO (2020a) World Health Organization. WHO draft landscape of Covid-19 candidate vaccines, Geneva. https://www.who.int/publications/m/item/draft-landscape-of-covid-19-candidate-vaccines. Access March 8 2021

16. James JJ (2020). Yin and Yang and Herd Immunity. Disaster medicine and public health preparedness. Cambridge University Press. https:// doi.org/10.1017/dmp.2020.229.

17. Fontanet A, Cauchemez S (2020) COVID-19 herd immunity: where are we? Nat Rev Immunol 20:583584. https://doi.org/10.1038/s41577-020-00451-5

18. Aguas R, Corder RM, King JG, Gonçalves G. Ferreira MU, Gomes GM (2020) Herd immunity thresholds for SARS-CoV-2 estimated from unfolding epidemics. https://doi.org/10.1101/2020.07.23.20160762 Access March 202021.

19. Seow J, Graham C, Acors S, Steel KJA, Hemmings O et al (2020) Longitudinal evaluation and decline of antibody responses in SARS-CoV-2 infection. https://doi.org/10.1101/2020.07.09.20148429

20. Ledford $H$ (2020) What the immune response to the coronavirus says about the prospects for a vaccine. Nature 585:20-21. https://doi.org/10.1038/d41586-020-02400-7

21. Mateus J, Grifoni A, Tarke A, Sidney J, Ramirez SI, Dan JM, Burger ZC, Rawlings SA, Smith DM, Phillips E, Mallal S, Lammers M, Rubiro P, Quiambao L, Sutherland A, Yu ED, da Silva Antunes R, Greenbaum J, Frazier A, Markmann AJ, Premkumar L, de Silva A, Peters B, Crotty S, Sette A, Weiskopf D (2020) Selective and cross-reactive SARS-CoV-2 T cell epitopes in unexposed humans. Science 370(6512):89-94

22. Possas C, Antunes AMS, Mendes FML, Schumacher SOR, Martins RM, Homma A et al (2015) Access to new technologies in multipatented vaccines: challenges for Brazil. Nat Biotechnol 33(6):599-603

23. WHO (2020b) World Health Organization. Ten threats to global health in 2019. Geneva

24. WHO (2020c) World Health Organization. Emergencies preparedness and response. Disease outbreak news (DONs), Geneva. https://www.who.int/csr/don/en/ 
25. WHO (2020d) World Health Organization. Public Health Emergency of International Concern. Statement on the second meeting of the International Health Regulations (2005) Emergency Committee regarding the outbreak of novel coronavirus (2019-nCoV). 30 January. Geneva.

26. Johns Hopkins University (2021) Coronavirus Resource Center. https://coronavirusjhuedu/maphtml access (February 28 2021)

27. UNAIDS, The Joint United Nations Programme on HIV/AIDS (2021) Global HIV \& AIDS statistics - 2020 fact sheet. Geneva https://wwwunaidsorg/sites/default/files/media_asset/UNAIDS_FactSheet_enpdf access April 212021

28. Reid A, Taubenberger J, Fanning T (2004) Evidence of an absence: the genetic origins of the 1918 pandemic influenza virus. Nat Rev Microbiol 2:909-914

29. Taubenberger J, Reid A, Lourens R et al (2005) Characterization of the 1918 influenza virus polymerase genes. Nature 437:889-893

30. Afrough B, Dowall S, Hewson R (2019) Emerging viruses and current strategies for vaccine intervention. Clin Exp Immunol 196:157-166

31. Jacobsen K (2020) Will COVID-19 generate global preparedness? Comment. Lancet 395(10229):10131014

32. Claas ECJ, Osterhaus ADME, Beek RV, Jong JCD, Rimmelzwaan GF, Senne DA et al (1998) Human influenza A H5N1 virus related to a highly pathogenic avian influenza virus. Lancet 351(9101):472-477

33. WHO (2020e) World Health Organization. Global Health Observatory World Health Statistics, Geneva. https://www.who.int/gho/publications/world_health_statistics/en/ access April 212021

34. Petersen LR, Jamieson DJ, Powers AM, Honein MA (2016) Zika Virus. N Engl J Med 21(374(16)):15521563

35. Possas C (2016) Zika: what we do and do not know based on the experiences of Brazil. Epidemiology and Health:e2016023

36. Possas C, Brasil P, Marzochi MC, Tanuri A, Martins RN, Marques ET et al (2017) Zika puzzle in Brazil: peculiar conditions of viral introduction and dissemination-a review. Mem Inst Oswaldo Cruz 112(5):319327

37. Coburn BJ, Wagner BG, Blower S (2009) Modeling influenza epidemics and pandemics: insights into the future of swine flu (H1N1). BMC Med 7:30

38. Feldmann MD, Geisbert TW (2011) Ebola haemorrhagic fever. Lancet 377((9768)):849-862

39. Leroy E, Kumulungui B, Pourrut X et al (2005) Fruit bats as reservoirs of Ebola virus. Nature 438:575-576

40. Azhar EI, El-Kafrawy SA, Farraj SA et al (2014) Evidence for camel-to-human transmission of MERS coronavirus. N Engl J Med 370:2499-2505

41. Yang Y, Du L, Liu C et al (2014) Receptor usage and cell entry of bat coronavirus HKU4 provide insight into bat-to-human transmission of MERS coronavirus. Proc Natl Acad Sci 111(34):12516-12521

42. Chinese SARS Molecular Epidemiology Consortium (2004) Molecular evolution of the SARS coronavirus during the course of the SARS epidemic in China. Science 303(5664):1666-1669

43. Peiris JSM, Chu CM, Cheng VCC, Chan KS, Hung IFN, Poon LLM, Law KI, Tang BS, Hon TY, Chan CS, Chan KH, Ng JS, Zheng BJ, Ng WL, Lai RW, Guan Y, Yuen KY, HKU/UCH SARS Study Group (2003) Clinical progression and viral load in a community outbreak of coronavirus-associated SARS pneumonia: a prospective study. Lancet 361(9371):1767-1772

44. Song Z, Xu Y, Bao L, Zhang L, Yu P, Qu Y, Zhu H, Zhao W, Han Y, Qin C (2019) From SARS to MERS, thrusting coronaviruses into the spotlight. Viruses (11):59

45. Grange ZL, Goldstein T, Johnson CK, Anthony S, Gilardi K, Daszak P et al (2021) Ranking the risk of animal-to-human spillover for newly discovered viruses PNAS 118

46. Nature Ecology and Evolution Editor (2020) Three-pronged pandemic prevention. Editorial Nat Ecol Evol 4:1149

47. Gibb R, Redding DW, Chin KQ, Donnelly CA, Blackburn TM, Newbold T, Jones KE (2020) Zoonotic host diversity increases in human-dominated ecosystems. Nature 584:398-302

48. Munnink BBO, Sikkema RS, Nieuwenhuijse DF, Molenar RJ, Munger E, Molenkamp R et al (2021) Transmission of SARS-CoV-2 on mink farms between humans and mink and back to humans Science. 371(6525):172-177. https://doi.org/10.1126/science.abe5901

49. Nuismer SL, Bull JJ (2020) Self-disseminating vaccines to suppress zoonoses. Nat Ecol Evol 4:1168-1173. https://doi.org/10.1038/s41559-020-1254-y

50. Murphy AA, Redwood AJ, Jarvis MA (2016) Self-disseminating vaccines for emerging infectious diseases. Expert Review of Vaccines 15(1):31-39

51. Kashyap H, Ahmed HA, Hoque N, Roy S, Bhattacharyya DK (2016) Big data analytics in bioinformatics: architectures, techniques, tools and issues. Netw Model Anal Health Inform Bioinforma 5:28 (2016). https:// doi.org/10.1007/s13721-016-0135-4 
52. Bowles J (2020) How Canadian AI start-up BlueDot spotted coronavirus before anyone else had a clue. Diginomica 1-2

53. BioSymetrics (2020) BioSymetrics collaborates with Janssen and Sema4 to predict the onset of COVID-19. http://wwwprnewswirecom/news-releases/biosymetrics-collaborates-with-janssen-and-sema4-to-predictthe-onset-of-covid-19-301114823html access April 82021.

54. Biovista (2020) Biovista harnesses new kinds of augmented and artificial intelligence to address covid-19. https://www.prnewswire.com/news-releases/biovista-harnesses-new-kinds-of-augmented-and-artificial-intelligence-to-address-covid-19-301116372.html Access April 182021.

55. Naudé W (2020) Artificial intelligence vs COVID-19: limitations, constraints and pitfalls. AI \& Soc 35: 761-765. https://doi.org/10.1007/s00146-020-00978-0

56. Gruber K (2017) Predicting zoonoses. Nat Ecol Evol 1:0098

57. Britton T, Ball F, Trapman P (2020) A mathematical model reveals the influence of population heterogeneity on herd immunity to SARS-CoV-2 Science.Jun $23 \mathrm{https} / /$ doi.org/10.1126/science.abc6810

58. ClinicalTrials.gov (2020) U.S. National Library of Medicine. Clinical Trials Database. https://clinicaltrials. gov/ Access October 4

\section{Affiliations}

\section{Cristina Possas $^{1}$ • Ernesto T. A. Marques ${ }^{2,3}$ • João Baptista Risi Jr ${ }^{1}$ • Akira Homma ${ }^{1}$}

Cristina Possas

cristina.possas@bio.fiocruz.br

1 Bio-Manguinhos, Oswaldo Cruz Foundation, Rio de Janeiro, Brazil

2 Aggeu Magalhães Institute, Oswaldo Cruz Foundation, Pernambuco, Brazil

3 Department of Infectious Diseases and Microbiology, University of Pittsburgh, Pittsburgh, USA 